Pacific Journal of Mathematic

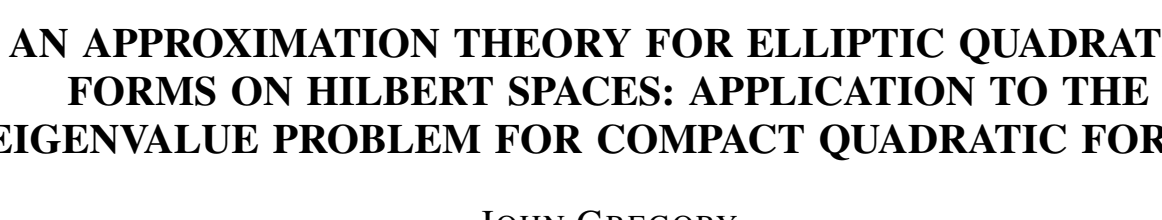




\title{
AN APPROXIMATION THEORY FOR ELLIPTIC QUADRATIC FORMS ON HILBERT SPACES: \\ APPLICATION TO THE EIGENVALUE PROBLEM FOR COMPACT QUADRATIC FORMS
}

\author{
JOHN GREGORY
}

A theory for an elliptic quadratic form $J(x)$ defined on a Hilbert space $\mathfrak{U}$ has been given by Hestenes. A fundamental part of this theory is concerned with the signature $s$ and nullity $n$ of $J(x)$ on $\mathfrak{A}$. These indices are used to develop a generalized Sturm-Lionville Theory and a Local Morse Theory. In this paper the theory of Hestenes is extended to elliptic quadratic forms $J(x ; \sigma)$ defined on $\mathfrak{N}(\sigma)$ where $\sigma$ is a member of the metric space $(\Sigma, \rho)$ and $\mathfrak{U}(\sigma)$ denotes a closed subspace of $\mathfrak{U}$. A fundamental part of this extension is concerned with inequalities dealing with the signature $s(\sigma)$ and nullity $n(\sigma)$ of $J(x ; \sigma)$ on $\mathfrak{U}(\sigma)$, where $\sigma$ is in a $\sigma$ neighborhood of a fixed point $\sigma_{0}$ in $\Sigma$.

It is noted that the hypothesis for these inequalities is sufficiently weak so as to include many mathematical problems. In the second part of this paper these results are applied to the study of eigenvalue problems for compact quadratic forms. A significant result is that the $n$th eigenvalue, $\lambda_{n}(\sigma)$, is a $\rho$ continuous function of $\sigma$. Comparison theorems are given for completeness. This work is a generalization of the eigenvalue theory of A. Weinstein.

The inequality results may also be used to study focal point problems and numerical approximation problems associated with linear self adjoint systems of ordinary or partial differential equations.

2. Preliminaries. The basic theory of Hilbert spaces, strong: and weak convergence, and linear operators and quadratic forms is given in References [2] and [3]. The fundamental Hilbert space is denoted by $\mathfrak{Y}$; subspaces by $\mathscr{B}, \mathscr{C}, \ldots$; elements of $\mathfrak{O}$ by the letters $x, y, z, \cdots$; scalars by $a, b, c, \cdots$. The inner product is denoted by $(x, y)$; the norm by $\|x\|$; strong convergence by $x_{q} \Rightarrow x_{0}$; weak convergence by $x_{q} \rightarrow x_{0}$. We will assume that subspaces of if are closed and the scalars are real. The latter assumption is for convenience; the complex case holds equally well.

A real valued function $L(x)$ defined on $\mathfrak{Q}$ is said to be a linear form if it is linear and continuous. A real valued function $Q(x, y)$ defined on $\mathfrak{A} \times \mathfrak{A}$ is a bilinear form if, for each $y$ in $\mathfrak{A}, Q(x, y)$ and 
$Q(y, x)$ are linear forms in $x$. If $x_{q} \rightarrow x_{0}$ and $y_{q} \rightarrow y_{0}$ imply $Q\left(x_{q}, y_{q}\right) \rightarrow$ $Q\left(x_{0}, y_{0}\right)$ then $Q(x, y)$ is compact. If $Q(x, y)=Q(y, x)$ then $Q(x)=$ $Q(x, x)$ is the quadratic form associated with the bilinear form $Q(x, y)$. We assume throughout this paper that bilinear forms satisfy $Q(x, y)=Q(y, x)$.

$Q(x)$ is positive (negative, nonpositive, nonegative) on $\mathfrak{A}$ if $Q(x)>0$ $(Q(x)<0, Q(x) \leqq 0, Q(x) \geqq 0)$ for $x \neq 0$ in $\mathrm{gr}^{\mathrm{r}} Q(x)$ is positive definite on $\mathfrak{A}$ if there exists a positive number $h$ such that $Q(x) \geqq h\|x\|^{2}$ on 2. $Q(x)$ is compact if $x_{q} \rightarrow x_{0}$ implies $Q\left(x_{q}\right) \rightarrow Q\left(x_{0}\right) . \quad Q(x)$ is weakly lower semicontinuous (wlsc) if $x_{q} \rightarrow x_{0}$ implies $\liminf _{q=\infty} Q\left(x_{q}\right) \geqq Q\left(x_{0}\right)$.

Two vectors $x$ any $y$ in $\mathfrak{A}$ are $Q$ orthogonal if $Q(x, y)=0$. The vector $x$ is $Q$ orthogonal to $\mathscr{B}$ if $y$ in $\mathscr{B}$ implies $Q(x, y)=0$. The set of all vectors $Q$ orthogonal to $\mathscr{B}$ is the $Q$ orthogonal complement, denoted by $\mathscr{B}^{Q} . \mathscr{B}$ and $\mathscr{C}$ are $Q$ orthogonal if each $x$ in $\mathscr{B}$ is $Q$ orthogonal to $\mathscr{C}$. A vector $x$ is a $Q$ null vector of $\mathscr{B}$ if $x$ in $\mathscr{B} \cap \mathscr{B}^{Q}$. $\mathscr{B}_{0}$ will denote the set of $Q$ null vectors of $\mathscr{B}$.

The signature (index) of $Q(x)$ on $\mathscr{B}$ is the dimension of a maximal, linear subclass $\mathscr{C}$ of $\mathscr{B}$ on which $Q(x)$ is negative. The nullity of $Q(x)$ on $\mathscr{B}$ is the dimension of $\mathscr{B}_{0}=\mathscr{B} \cap \mathscr{B}^{Q}$. Finally $J(x)$ is an elliptic form on $\mathfrak{A}$ if $J(x)$ is wlsc on $\mathfrak{A}$, and $x_{q} \Rightarrow x_{0}$ whenever $x_{q} \rightarrow x_{0}$ and $J\left(x_{q}\right) \rightarrow J\left(x_{0}\right)$.

We note the following results for elliptic forms [3]: A quadratic form $J(x)$ is elliptic on $\mathfrak{A}$ if and only if there exists a finite dimensional subspace $\mathscr{B}$ of $\mathfrak{A}$ such that $J(x)$ is positive definite on the orthogonal complement of $\mathscr{B}$. A quadratic form $J(x)$ is elliptic on $\mathfrak{A}$ if and only if there exists a positive definite form $P(x)$ and a compact form $K(x)$ such that $J(x)=P(x)-K(x)$. Furthermore $K(x)$ can be chosen nonnegative on $\mathfrak{A}$. A positive elliptic form is positive definite.

Theorems 1 and 2 have been given in [3].

THEOREM 1. The signature of $Q(x)$ on $\mathscr{B}$, if finite, is given by each of the following quantities:

(a) the dimension of a maximal subspase $\mathscr{C}$ in $\mathscr{B}$ on which $Q(x)<0$;

(b) the dimension of a maximal subspase $\mathscr{D}$ in $\mathscr{B}$ on which $Q(x) \leqq 0$ and $\mathscr{D} \cap \mathscr{B}_{0}=0$;

THEOREM 2. If the sum $m=s+n$ of the signature $s$ and nullity $n$ of $Q(x)$ on $\mathscr{B}$ is finite, it is given by each of the following quantities:

(a) the dimension of a maximal subspace $\mathscr{C}$ in $\mathscr{B}$ in which $Q(x) \leqq 0$; 
(b) the least integer $k$ such that there exists $k$ linear forms $L_{1}, \cdots, L_{k}$ on $\mathscr{B}$ with $Q(x)>0$ for all $x$ in $\mathscr{B}$ satisfying $L_{\alpha}(x)=$ $0(\alpha=1, \cdots, k)$.

3. Fundamental Inequalities. The purpose of this section is to state and derive fundamental inequalities which relate the signature and nullity of an Elliptic Form on a closed subspace of $\mathfrak{A}$ to "approximating" Elliptic Forms on "approximating" closed subspaces.

The main results are contained in Theorems 6 and 7 . Theorem 8 is a combination of these two theorems. Theorem 11 is an extension of Theorem 7 to the metric space $M=E^{1} \times \Sigma$. Continuity of the $n$th eigenvalue, $\lambda_{n}(\sigma)$, follows immediately from Theorem 11 .

Let $\Sigma$ be a metric space with metric $\rho$. A sequence $\left\{\sigma_{r}\right\}$ in $\Sigma$ converges to $\sigma_{0}$ in $\Sigma$, written $\sigma_{r} \rightarrow \sigma_{0}$, if $\lim _{r=\infty} \rho\left(\sigma_{r}, \sigma_{0}\right)=0$. For each $\sigma$ in $\Sigma$ let $\mathfrak{U}(\sigma)$ be a closed subspace of $\mathfrak{U}$ such that

(1a) If $\sigma_{r} \rightarrow \sigma_{0}, x_{r}$ in $\mathfrak{A}\left(\sigma_{r}\right), x_{r} \rightarrow y_{0}$ then $y_{0}$ is in $\mathfrak{A}\left(\sigma_{0}\right)$;

(1b) If $x_{0}$ is in $\mathfrak{U}\left(\sigma_{0}\right)$ and $\varepsilon>0$ there exists $\delta>0$

such that whenever $\rho\left(\sigma, \sigma_{0}\right)<\delta$, there exists $x_{\sigma}$ in $\mathfrak{U}(\sigma)$ satisfying $\left\|x_{0}-x_{\sigma}\right\|<\varepsilon$.

LEMMA 3. Condition (1b) is equivalent to the following: Let $\mathscr{B}\left(\sigma_{0}\right)$ be a subspace of $\mathfrak{U}\left(\sigma_{0}\right)$ of dimension $h$ and $\varepsilon>0$. There exists $\delta>0$ such that whenever $\rho\left(\sigma_{0}, \sigma\right)<\delta$, there exists a subspace. $\mathscr{B}(\sigma)$ of $\mathfrak{H}(\sigma)$ of dimension $h$ with the property that if $x_{0}$ is a unit vector in $\mathscr{B}\left(\sigma_{0}\right)$ there exists $x_{\sigma}$ in $\mathscr{B}(\sigma)$ such that $\left\|x_{0}-x_{\sigma}\right\|<\varepsilon$.

Clearly this condition implies (1b) with $h=1$. Conversely let $x_{1}, \cdots, x_{h}$ be an orthonormal basis for $\mathscr{B}\left(\sigma_{0}\right)$. Given $\varepsilon>0$ there exists $\delta>0$ such that if $\rho\left(\sigma_{0}, \sigma\right)<\delta$ then $x_{1 \sigma}, \cdots, x_{h \sigma}$ is in $\mathfrak{U}(\sigma)$ with $\left\|x_{k}-x_{k \sigma}\right\|^{2}<\varepsilon / h$.

Assume that usual summation conventions with $k, l=1, \cdots, h$. Letting $x_{0}=b_{k} x_{k}$ and $x_{\sigma}=b_{k} x_{k \sigma}$ where $b_{k} b_{k}=1$ we have

$$
\begin{aligned}
\left\|x_{0}-x_{\sigma}\right\|^{2} & =\left\|b_{k}\left(x_{k}-x_{k \sigma}\right)\right\|^{2} \leqq\left(\left|b_{k}\right|\left\|x_{k}-x_{k \sigma}\right\|\right)^{2} \\
& \leqq\left(b_{k} b_{k}\right)\left(\left\|x_{l}-x_{l \sigma}\right\|\left\|x_{l}-x_{l \sigma}\right\|\right) \leqq h(\varepsilon / h)=\varepsilon
\end{aligned}
$$

This concludes the proof of the lemma.

The "approximation" hypothesis for forms are now stated.

For each $\sigma$ in $\Sigma$ let $J(x ; \sigma)$ be a quadratic form defined on $\mathfrak{Y}(\sigma)$ with $J(x, y ; \sigma)$ the associated bilinear form. Let $s(\sigma)$ and $n(\sigma)$ be the index and nullity of $J(x ; \sigma)$ on $\mathfrak{A}(\sigma)$. For $r=0,1,2, \cdots$ let $x_{r}$ be in $\mathfrak{U}\left(\sigma_{r}\right), y_{r}$ in $\mathfrak{U}\left(\sigma_{r}\right)$ such that: if $x_{r} \rightarrow x_{0}, y_{r} \Rightarrow y_{0}$ and $\sigma_{r} \rightarrow \sigma_{0}$ then 
(2a) $\lim _{r=\infty} J\left(x_{r}, y_{r} ; \sigma_{r}\right)=J\left(x_{0}, y_{0} ; \sigma_{0}\right)$;

(2b) $\quad \lim \inf J\left(x_{r} ; \sigma_{r}\right) \geqq J\left(x_{0} ; \sigma_{0}\right)$; and

(2c) $\lim _{r=\infty} J\left(x_{r} ; \sigma_{r}\right)=J\left(x_{0} ; \sigma_{0}\right)$ implies $x_{r} \Rightarrow x_{0}$.

Lemma 4. Assume condition (2a) holds. Let $\sigma_{0}$ be given. Then there exists $\delta>0, M>0$ sush that $\rho\left(\sigma, \sigma_{0}\right)<\delta$ implies $|J(x, y ; \sigma)| \leqq$ $M\|x\|\|y\|$ for all $x, y$ in $\mathfrak{Q}(\sigma)$.

Suppose the conclusion does not hold. Then for $r=1,2, \cdots$ we may choose $\sigma_{r}$ in $\Sigma$ and $x_{r}, y_{r}$ in $\mathfrak{P}\left(\sigma_{r}\right)$ such that $\left\|x_{r}\right\|=\left\|y_{r}\right\|=1$, $\rho\left(\sigma_{r}, \sigma_{0}\right)<1 / r$ and $a_{r}^{2}=\left|J\left(x_{r}, y_{r} ; \sigma_{r}\right)\right|>r$.

Now $\bar{x}_{r}=x_{r} / a_{r} \Rightarrow 0$ and $\bar{y}_{r}=y_{r} / a_{r} \Rightarrow 0$ so by

$$
1=J\left(\bar{x}_{r}, \bar{y}_{r} ; \sigma_{r}\right) \longrightarrow J\left(0,0 ; \sigma_{0}\right)=0 .
$$

This contradiction establishes the result.

THEOREM 5. If (2a) and (2c) hold then either $J(x ; \sigma)$ or $-J(x ; \sigma)$ satisfy (2b).

Suppose the conclusion does not hold. Then there exists sequences $\left\{\sigma_{r}\right\},\left\{y_{r}\right\}$ and $\left\{z_{r}\right\} \quad(r=0,1,2, \cdots)$ such that $\sigma_{r} \rightarrow \sigma_{0} ; y_{r}, z_{r}$ in $\mathfrak{R}\left(\sigma_{r}\right)$; $y_{r} \rightarrow y_{0}, z_{r} \rightarrow z_{0}$; and

$$
\begin{aligned}
& \lim _{r=\infty} J\left(y_{r} ; \sigma_{r}\right)=A<J\left(y_{0} ; \sigma_{0}\right), \\
& \lim _{r=\infty} J\left(y_{r}, z_{r} ; \sigma_{r}\right)=B, \text { and } \\
& \lim _{r=\infty} J\left(z_{r} ; \sigma_{r}\right)=C>J\left(z_{0} ; \sigma_{0}\right)
\end{aligned}
$$

where $A, B$, and $C$ are real numbers by Lemma 4 . Thus the equation

$$
\left[A-J\left(y_{0} ; \sigma_{0}\right)\right] \alpha^{2}+2 \alpha\left[B-J\left(y_{0}, z_{0} ; \sigma_{0}\right)\right]+\left[C-J\left(z_{0} ; \sigma_{0}\right)\right]=0
$$

has two distinct real roots $a_{1}, a_{2}$. For $i=1,2$ and $r=0,1,2, \cdots$ let $x_{r i}=a_{i} y_{r}+z_{r}$ so that $x_{r i} \rightarrow x_{0 i}$. By the definition of $a_{i}$,

$$
\begin{aligned}
J\left(x_{r i} ; \sigma_{r}\right) & =J\left(y_{r} ; \sigma_{r}\right) a_{i}^{2}+2 a_{i} J\left(y_{r}, z_{r} ; \sigma_{r}\right)+J\left(z_{r} ; \sigma_{r}\right) \longrightarrow A a_{i}^{2}+2 B a_{i}+C \\
& =J\left(y_{0} ; \sigma_{0}\right) a_{i}^{2}+2 a_{i} J\left(y_{0}, z_{0} ; \sigma_{0}\right)+J\left(z_{0} ; \sigma_{0}\right)=J\left(x_{0 i} ; \sigma_{0}\right)
\end{aligned}
$$

so that from (2c) $x_{r i} \Rightarrow x_{0 i}(i=1,2)$. Since $a_{1} \neq a_{2}$ then $y_{q} \Rightarrow y_{0}$ and $z_{q} \Rightarrow z_{0}$. Finally from (2a) we have

$$
A=\lim _{r=\infty} J\left(y_{r} ; \sigma_{r}\right)=J\left(y_{0} ; \sigma_{0}\right)>A .
$$

This contradiction establishes the theorem.

Theorem 6. Assume conditions (1a), (2b) and (2c) hold. Then 
for any $\sigma_{0}$ in $\Sigma$ there exists $\delta>0$ such that $\rho\left(\sigma_{0}, \sigma\right)<\delta$ implies

$$
s(\sigma)+n(\sigma) \leqq s\left(\sigma_{0}\right)+n\left(\sigma_{0}\right)
$$

Assume the conclusion is false. Then there exists a sequence $\left\{\sigma_{r}\right\}$ with $\sigma_{r} \rightarrow \sigma_{0}$ and $s\left(\sigma_{r}\right)+n\left(\sigma_{r}\right)>s\left(\sigma_{0}\right)+n\left(\sigma_{0}\right)$. Let $k=s\left(\sigma_{0}\right)+n\left(\sigma_{0}\right)+1$. For $r=1,2, \cdots$ there exists $k$ orthonormal vectors $x_{1 r}, x_{2 r}, \cdots, x_{k r}$ in $\mathfrak{U}\left(\sigma_{r}\right)$ with $J\left(x ; \sigma_{r}\right) \leqq 0$ on $\operatorname{span}\left\{x_{1 r}, \cdots, x_{k r}\right\}$. For each $p=1, \cdots, k$ the sequence $\left\{x_{p r}\right\}$ is bounded in $\mathfrak{Y}$ and hence has a weakly convergent subsequence, which we may assume to be $\left\{x_{p r}\right\}$, such that $x_{p r} \rightarrow x_{p}$. By (1a) $x_{p}$ is in $\mathfrak{U}\left(\sigma_{0}\right)$.

Assume the usual repeated index summation convention with $p=1, \cdots, k$. Let $b=\left(b_{1}, \cdots, b_{k}\right)$ be arbitrary, set $y_{0}=b_{p} x_{p}$ and $y_{r}=b_{p} x_{p r}$. Since $y_{r} \rightarrow y_{0}$ we have by $(2 \mathrm{~b})$

$$
J\left(y_{0} ; \sigma_{0}\right) \leqq \liminf _{r=\infty} J\left(y_{r} ; \sigma_{r}\right) \leqq 0
$$

Thus $x_{1}, \cdots, x_{k}$ is a linear dependent set, for if not by Theorem 2, $k-1=s\left(\sigma_{0}\right)+n\left(\sigma_{0}\right) \geqq k$.

Choose $b \neq 0$ such that $y_{0}=b_{p} x_{p}=0$; also choose $y_{r}=b_{p} x_{p r}$. We note $y_{r} \rightarrow y_{0}=0$ and

$$
0=J\left(0 ; \sigma_{0}\right) \leqq \liminf _{r=\infty} J\left(y_{r} ; \sigma_{r}\right) \leqq \limsup _{r=\infty} J\left(y_{r} ; \sigma_{r}\right) \leqq 0 .
$$

Hence $J\left(y_{r} ; \sigma_{r}\right) \rightarrow 0=J\left(0 ; \sigma_{0}\right)$ so that $y_{r} \Rightarrow 0$ by $(2 \mathrm{c})$.

Finally $0=\lim _{r=\infty}\left\|y_{r}\right\|^{2}=b_{p} b_{p} \neq 0$. This contradiction establishes the theorem.

THEOREM 7. Assume conditions (1b) and (2a) hold. Then for any $\sigma_{0}$ in $\Sigma$ there exists $\delta>0$ such that $\rho\left(\sigma_{0}, \sigma\right)<\delta$ implies

$$
s\left(\sigma_{0}\right) \leqq s(\sigma)
$$

Let $\mathscr{B}\left(\sigma_{0}\right)$ be a maximal subspace of $\mathfrak{H}\left(\sigma_{0}\right)$ such that $J\left(x ; \sigma_{0}\right)<0$ on $\mathscr{B}\left(\sigma_{0}\right)$. Let $x_{1}, \cdots, x_{h}$ be a basis for $\mathscr{B}\left(\sigma_{0}\right)$. By Lemma 3 and conditions (1b) and (2a) there exists a basis $x_{1 \sigma}, \cdots, x_{h \sigma}$ for $\mathscr{B}(\sigma)$ such that if $x_{\sigma}=a_{p} x_{p \sigma}$ and

$$
A_{p q}(\sigma)=J\left(x_{p \sigma}, x_{q \sigma} ; \sigma\right)
$$

then

$$
F(a, \sigma)=J\left(x_{\sigma} ; \sigma\right)=a_{p} a_{q} A_{p q}(\sigma) \quad(p, q=1, \cdots, h ; p, q \text { summed })
$$

is a continuous function of $\sigma$ at $\sigma_{0}$.

By the usual arguments for quadratic forms we may choose $M<0$ and $\delta>0$ such that 


$$
F\left(a, \sigma_{0}\right) \leqq 2 M a_{p} a_{p}
$$

and

$$
F(a, \sigma)=F\left(a, \sigma_{0}\right)+\left(A_{p q}(\sigma)-A_{p q}\left(\sigma_{0}\right)\right) a_{p} a_{q} \leqq M a_{p} a_{p}
$$

where $\rho\left(\sigma_{0}, \sigma\right)<\delta$. This completes the proof.

Combining Theorems 6 and 7 we obtain

THEOREM 8. Assume conditions (1) and (2) hold. Then for any $\sigma_{0}$ in $\Sigma$ there exists $\delta>0$ such that $\rho\left(\sigma, \sigma_{0}\right)<\delta$ implies

$$
s\left(\sigma_{0}\right) \leqq s(\sigma) \leqq s(\sigma)+n(\sigma) \leqq s\left(\sigma_{0}\right)+n\left(\sigma_{0}\right) .
$$

Corollary 9. Assume $\delta>0$ has been choosen such that $\rho\left(\sigma, \sigma_{0}\right)<$ $\delta$ implies equation (5) holds. Then if $\rho\left(\sigma, \sigma_{0}\right)<\delta$ we have

(6a) $n(\sigma) \leqq n\left(\sigma_{0}\right)$

(6b) $n(\sigma)=n\left(\sigma_{0}\right)$ implies $s(\sigma)=s\left(\sigma_{0}\right)$ and $m(\sigma)=m\left(\sigma_{0}\right)$, and

(6c) $n\left(\sigma_{0}\right)=0$ implies $s(\sigma)=s\left(\sigma_{0}\right)$ and $n(\sigma)=0$.

This result follows at once from Theorem 8. As a further result we have

COROLlaRY 10. The set $\{\sigma \in \Sigma \mid n(\sigma)=0\}$ is open. The set $\{\sigma \in \Sigma \mid n(\sigma) \neq 0\}$ is closed.

As an example of these results we will extend Theorem 8 to a result for the metric space $M=E^{1} \times \Sigma$. This result will be fundamental for the continuity of the $n$th eigenvalue $\lambda^{n}(\sigma)$. Thus assume $M=I \times \Sigma$, I an open interval of $E^{1}$, is a metric space with metric $d$ defined by

$$
d\left(\mu_{1}, \mu_{2}\right)=\left|\lambda_{2}-\lambda_{1}\right|+\rho\left(\sigma_{2}, \sigma_{1}\right)
$$

for any pair of points $\mu_{1}=\left(\lambda_{1}, \sigma_{1}\right), \mu_{2}=\left(\lambda_{2}, \sigma_{2}\right)$ in $M$. Let $s(\mu)=$ $s(\lambda, \sigma), n(\mu)=n(\lambda, \sigma)$ be the index and nullity of $J(x ; \mu)$ on $\mathfrak{A}(\mu)$; let $m(\mu)=m(\lambda, \sigma)=s(\lambda, \sigma)+n(\lambda, \sigma)$. Theorem 8 and Corollary 9 hold with the obvious modifications.

THEOREM 11. Let conditions (1), (2) be satisfied with $\mu=(\lambda, \sigma)$ in $M$ replacing $\sigma$ in $\Sigma$. For fixed $\sigma$ let the signature $s(\lambda, \sigma)$ be a monotone function of $\lambda$ such that $s(\lambda+0, \sigma)=s(\lambda-0, \sigma)$ implies $n(\lambda, \sigma)=0$. Let $\mu_{0}=\left(\lambda_{0}, \sigma_{0}\right)$ in $M$ be given such that $s\left(\lambda_{0}-0, \sigma_{0}\right)=$ $n, s\left(\lambda_{0}+0, \sigma_{0}\right)=m$. Then there exists $\delta_{0}>0$ such that $\left|\lambda-\lambda_{0}\right| \leqq \delta_{0}$ and $\rho\left(\sigma, \sigma_{0}\right) \leqq \delta_{0}$ imply that $s(\lambda, \sigma)$ is between $n$ and $m$.

Assume $s(\lambda, \sigma)$ is monotone increasing on an interval $I$ and 
hence $n \leqq m$. Choose $\delta>0$ so small that $s\left(\lambda, \sigma_{0}\right)=n$ for $\left(\lambda_{0}-2 \delta, \lambda_{0}\right) \subset I$ and $s\left(\lambda, \sigma_{0}\right)=m$ for $\left(\lambda_{0}, \lambda_{0}+2 \delta\right) \subset I$. By assumption $n\left(\lambda_{0}-\delta, \sigma_{0}\right)=$ $n\left(\lambda_{0}+\delta, \sigma_{0}\right)=0$. Finally choose $\delta_{0}, 0<\delta_{0} \leqq \delta$, such that $\rho\left(\sigma, \sigma_{0}\right)<\delta_{0}$ implies (5) holds for both $\mu_{0}=\left(\lambda_{0}-\delta, \sigma_{0}\right)$ and $\mu_{0}=\left(\lambda_{0}+\delta, \sigma_{0}\right)$. By (6c), $s\left(\lambda_{0}-\delta, \sigma\right)=n$ and $s\left(\lambda_{0}+\delta, \sigma\right)=m$ for all $\sigma$ such that $\rho\left(\sigma, \sigma_{0}\right)<\delta_{0}$. The theorem now follows by the monotone condition.

4. Eigenvalue Theory. The purpose of this section is to apply the theory of $\S 3$ to the study of eigenvalue problems for compact quadratic forms. Our work is motivated by (and at times duplicates) the methods and results of Hestenes [3]. Of particular significance are Theorems 20 and 21 which give sufficiency conditions for the continuity of the $n$th eigenvalue. Theorem 22 and 23 are comparison theorems. They follow directly from "signature inequalities" given in Reference [3].

In this section we assume $\Sigma$ is a metric space with metric $\rho$. For each $\sigma$ in $\Sigma$ let $\mathfrak{A}(\sigma)$ be a closed subspace of $\mathfrak{A}, J(x ; \sigma)$ an elliptic form defined on $\mathfrak{A}(\sigma)$, and $K(x ; \sigma)$ a compact form on $\mathfrak{A}(\sigma)$.

We assume conditions (1) and (2) are satisfied and that $\sigma_{r} \rightarrow \sigma_{0}$, $x_{r}$ in $\mathfrak{A}\left(\sigma_{r}\right), x_{0}$ in $\mathfrak{A}\left(\sigma_{0}\right), x_{r} \rightarrow x_{0}$ imply $K\left(x_{r} ; \sigma_{r}\right) \rightarrow K\left(x_{0} ; \sigma_{0}\right)$.

Let $M=E^{1} \times \Sigma$ be the metric space with metric $d$ defined above (after Corollary 10). For each $\mu=(\lambda, \sigma)$ in $M$ define $\mathfrak{A}(\mu)=\mathfrak{A}(\sigma)$ and

$$
H(x ; \mu)=J(x ; \lambda, \sigma)=J(x ; \sigma)-\lambda K(x ; \sigma)
$$

on the space $\mathfrak{U}(\mu)$. Finally let $s(\mu)=s(\lambda, \sigma), n(\mu)=n(\lambda, \sigma)$, and $m(\mu)=m(\lambda, \sigma)$ denote the index, nullity, and sum of the index and nullity of $H(x ; \mu)$ on $\mathfrak{A}(\mu)$.

THEOREM 12. Conditions (1) and (2) hold with $\mu$ replacing $\sigma$ and $H$ replacing $J$.

Since $\mathfrak{A}(\mu)=\mathfrak{A}(\sigma)$ conditions (1) hold. For (2a) let $x_{r}, y_{r}$ in $\mathfrak{A}\left(\mu_{r}\right), r=0,1,2, \cdots$ with $x_{r} \rightarrow x_{0}$ and $y_{r} \Rightarrow y_{0}$. Then

$$
\begin{aligned}
H\left(x_{r}, y_{r} ; \mu_{r}\right)-H\left(x_{0}, y_{0} ; \mu_{0}\right)= & \left\{\left(x_{r}, y_{r} ; \sigma_{r}\right)-J\left(x_{0}, y_{0} ; \sigma_{0}\right)\right\} \\
& +\lambda_{0}\left[K\left(x_{0}, y_{0} ; \sigma\right)-K\left(x_{r}, y_{r} ; \sigma_{r}\right)\right] \\
& +\left(\lambda_{0}-\lambda_{r}\right) K\left(x_{r}, y_{r} ; \sigma_{r}\right) .
\end{aligned}
$$

If $r \rightarrow \infty$ the first term goes to 0 since (2) holds on $\Sigma$, the third term goes to 0 as $K\left(x_{r}, y_{r} ; \sigma_{r}\right)$ is bounded, and the second term goes to 0 by the equality 


$$
\begin{aligned}
2\left[K\left(x_{0}, y_{0} ; \sigma_{0}\right)-K\left(x_{r}, y_{r} ; \sigma_{r}\right)\right]= & K\left(x_{0}+y_{0} ; \sigma_{0}\right)-K\left(x_{r}+y_{r} ; \sigma_{r}\right) \\
& -K\left(x_{0} ; \sigma_{0}\right)-K\left(y_{0} ; \sigma_{0}\right) \\
& +K\left(x_{r} ; \sigma_{r}\right)+K\left(y_{r} ; \sigma_{r}\right) .
\end{aligned}
$$

For $(2 \mathrm{~b})$ let $\underline{\lim } A_{r}$ denote $\lim \inf A_{r}$ then

$$
\begin{aligned}
\underline{\lim H\left(x_{r} ; \mu_{r}\right)} & =\underline{\lim }\left[J\left(x_{r} ; \sigma_{r}\right)-\lambda_{r} K\left(x_{r} ; \sigma_{r}\right)\right] \\
& \geqq \underline{\lim } J\left(x_{r} ; \sigma_{r}\right)-\lim _{r=\infty} \lambda_{r} K\left(x_{r} ; \sigma_{r}\right) \geqq J\left(x_{0} ; \sigma_{0}\right)-\lambda_{0} K\left(x_{0} ; \sigma_{0}\right) \\
& =H\left(x_{0} ; \mu_{0}\right) .
\end{aligned}
$$

For $(2 \mathrm{c})$ if $x_{r} \rightarrow x_{0}, \lim H\left(x_{r} ; \mu_{r}\right)=H\left(x_{0} ; \mu_{0}\right)$ then

$$
\begin{aligned}
J\left(x_{0} ; \sigma_{0}\right)-\lambda_{0} K\left(x_{0} ; \sigma_{0}\right)= & H\left(x_{0} ; \mu_{0}\right)=\lim _{r=\infty} H\left(x_{r} ; \mu_{r}\right)=\lim _{r=\infty} J\left(x_{r} ; \sigma_{r}\right) \\
& -\lim _{r=\infty} \lambda_{r} K\left(x_{r} ; \sigma_{r}\right)
\end{aligned}
$$

so that $J\left(x_{0} ; \sigma_{0}\right)=\lim _{r=\infty} J\left(x_{r} ; \sigma_{r}\right)$. Since (2c) holds on $\Sigma$, we have $x_{r} \Rightarrow x_{0}$. This complete the proof of the theorem.

Theorem 13 now follows immediately from Theorem 8 .

THEOREM 13. For any $\mu_{0}=\left(\lambda_{0}, \sigma_{0}\right)$ in $M$ there exists $\delta>0$ such that if $\mu=(\lambda, \sigma), d\left(\mu, \mu_{0}\right)<\delta$ then

(8) $\quad s\left(\lambda_{0}, \sigma_{0}\right) \leqq s(\lambda, \sigma) \leqq s(\lambda, \sigma)+n(\lambda, \sigma) \leqq s\left(\lambda_{0}, \sigma_{0}\right)+n\left(\lambda_{0}, \sigma_{0}\right)$.

Corollary 14. Assume $\delta>0$ has been chosen such that $\mu=$ $(\lambda, \sigma), d\left(\mu, \mu_{0}\right)<\delta$ implies inequalities (8) hold. Then if $d\left(\mu, \mu_{0}\right)<\delta$ we have

(9a) $n(\lambda, \sigma) \leqq n\left(\lambda_{0}, \sigma_{0}\right)$,

(9b) $n(\lambda, \sigma)=n\left(\lambda_{0}, \sigma\right)$ implies $s(\lambda, \sigma)=s\left(\lambda_{0}, \sigma_{0}\right)$ and $m(\lambda, \sigma)=m\left(\lambda_{0}, \sigma_{0}\right)$, and

(9c) $n\left(\lambda_{0}, \sigma_{0}\right)=0$ implies $s(\lambda, \sigma)=s\left(\lambda_{0}, \sigma_{0}\right)$ and $n(\lambda, \sigma)=0$.

Corollaries 14 and 15 follow immediately from Theorem 13.

Corollary 15. The set $\{\mu$ in $M \mid n(\mu)=0\}$ is open. The set $\{\mu$ in $M \mid n(\mu) \neq 0\}$ is closed.

THEOREM 16. Let $\sigma_{0}$ in $\Sigma$ be given and let $\Lambda_{0}$ be a nonempty compact subset of $\left\{\lambda \mid n\left(\lambda, \sigma_{0}\right)=0\right\}$. Then there exists $\varepsilon>0$ such that $\lambda_{0}$ in $\Lambda_{0 \varepsilon}$ and $\rho\left(\sigma, \sigma_{0}\right)<\varepsilon$ imply

$$
s\left(\lambda_{0}, \sigma\right)=s\left(\lambda_{0}, \sigma_{0}\right), \quad n\left(\lambda_{0}, \sigma\right)=n\left(\lambda_{0}, \sigma_{0}\right)=0
$$

where $\Lambda_{0 \varepsilon}$ is the $\varepsilon$-neighborhood of $\Lambda_{0}$.

Let $\lambda_{0}$ in $\Lambda_{0}$ and set $\mu_{0}=\left(\lambda_{0}, \sigma_{0}\right)$. By Corollary 14 there exists $\delta=\delta\left(\lambda_{0}\right)>0$ such that $\mu=(\lambda, \sigma)$ and $d\left(\mu, \mu_{0}\right)<\delta$ imply conditions 
(10). By the usual arguments for compact sets there exists $\delta>0$ such that (10) holds whenever $d\left(\mu, \mu_{0}\right)<\delta$ for any $\lambda_{0}$ in $\Lambda_{0}$. This completes the proof.

COROLlary 17. Let $\lambda^{*}$ be real and $\sigma_{0}$ in $\Sigma$ such that $n\left(\lambda^{*}, \sigma_{0}\right)=$ $s\left(\lambda^{*}, \sigma_{0}\right)=0$. Then there exists $\varepsilon>0$ such that $\rho\left(\sigma, \sigma_{0}\right)<\varepsilon$ and $\left|\lambda-\lambda_{0}\right|<\varepsilon$ imply $n(\lambda, \sigma)=s(\lambda, \sigma)=0$.

Let $\sigma_{0}$ in $\Sigma$ be given. A real number $\lambda_{0}$ is an eigenvalue (characteristic value) of $J\left(x ; \sigma_{0}\right)$ relative to $K\left(x ; \sigma_{0}\right)$ on $\mathfrak{T}\left(\sigma_{0}\right)$ if $n\left(\lambda_{0}, \sigma_{0}\right) \neq 0$. The number $n\left(\lambda_{0}, \sigma_{0}\right)$ is its multiplicity. An eigenvalue $\lambda_{0}$ will be counted the number of times equal to its multiplicity. If $\lambda_{0}$ is an eigenvalue and $x_{0} \neq 0$ in $\mathfrak{Y}\left(\sigma_{0}\right)$ such that $J\left(x_{0}, y ; \sigma_{0}\right)=$ $\lambda_{0} K\left(x_{0}, y ; \sigma_{0}\right)$ for all $y$ in $\mathfrak{H}\left(\sigma_{0}\right)$ then $x_{0}$ is an eigenvector corresponding to $\lambda_{0}$.

Assume $J, K$, and $\mathfrak{2}$ are independent of $\sigma$, that is, consider a fixed Elliptic Form $J(x)$ and a fixed compact form $K(x)$ on a fixed space $\mathfrak{A}$. Results for this case (Theorem 18) have been given by Hestenes [3].

THEOREM 18. Assume $x \neq 0$ in $\mathfrak{N}, K(x) \leqq 0$ implies $J(x)>0$. Then there exists $\lambda^{*}$ such that $J\left(x ; \lambda^{*}\right)$ is positive definite on $\mathfrak{X}$.

If $\lambda_{0} \geqq \lambda^{*}$ there exists $\varepsilon=\varepsilon\left(\lambda_{0}\right)$ sush that

(11a) $s(\lambda)=s\left(\lambda_{0}\right), n(\lambda)=0\left(\lambda_{0}-\varepsilon<\lambda<\lambda_{0}\right)$ and

(11b) $s(\lambda)=s\left(\lambda_{0}\right)+n\left(\lambda_{0}\right), n(\lambda)=0\left(\lambda_{0}<\lambda<\lambda_{0}+\varepsilon\right)$.

If $\lambda_{0} \leqq \lambda^{*}$ there exists $\varepsilon=\varepsilon\left(\lambda_{0}\right)$ such that

(12a) $s(\lambda)=s\left(\lambda_{0}\right)+n\left(\lambda_{0}\right), n(\lambda)=0\left(\lambda-\varepsilon<\lambda<\lambda_{0}\right)$ and

(12b) $s(\lambda)=s\left(\lambda_{0}\right), n(\lambda)=0\left(\lambda_{0}<\lambda<\lambda_{0}+\varepsilon\right)$.

If $\lambda^{*} \leqq \lambda^{\prime}<\lambda^{\prime \prime}$ then $s\left(\lambda^{\prime \prime}\right)-s\left(\lambda^{\prime}\right)$ is equal to the number of characteristic values on $\lambda^{\prime} \leqq \lambda<\lambda^{\prime \prime}$; if $\lambda^{\prime \prime}<\lambda^{\prime} \leqq \lambda^{*}$ then $s\left(\lambda^{\prime \prime}\right)-s\left(\lambda^{\prime}\right)$ is equal to the number of characteristic values on $\lambda^{\prime \prime}<\lambda \leqq \lambda^{\prime}$.

If $\lambda^{*} \leqq \lambda^{\prime}<\lambda^{\prime \prime}$ then $s\left(\lambda^{\prime \prime}\right)+n\left(\lambda^{\prime \prime}\right)-s\left(\lambda^{\prime}\right)$ is equal to the number of charasteristic values on $\lambda^{\prime} \leqq \lambda \leqq \lambda^{\prime \prime}$; if $\lambda^{\prime \prime}<\lambda^{\prime} \leqq \lambda^{*}$ then $s\left(\lambda^{\prime \prime}\right)+$ $n\left(\lambda^{\prime \prime}\right)-s\left(\lambda^{\prime}\right)$ is exual to the number of charasteristis values on $\lambda^{\prime \prime} \leqq \lambda \leqq \lambda^{\prime}$.

It is instructive to describe the graph of $\lambda$ versus $s(\lambda)$. By Theorem 18 this graph is a step function with a finite or countably infinite number of intervals; each interval has the associated nonnegative integer value $s(\lambda)$. The number $\lambda^{*}$ is not unique. It may be chosen to be any interior point of the interval on which $s(\lambda)=0$. Note that $s(\lambda)$ is a nondecreasing function on $\left(\lambda^{*}, \infty\right)$ and nonincreasing on $\left(-\infty, \lambda^{*}\right)$; it is continuous from the right if $\lambda<\lambda^{*}$ and from the left it $\lambda^{*}<\lambda$. The discontinuities in $s(\lambda)$ are points at which $n(\lambda) \neq 0$; in fact the jump at $\lambda$ is $n(\lambda)$. 
For convenience we will denote the $k$ th eigenvalue greater than $\lambda^{*}$ by $\lambda_{k-1}$, the $k$ th eigenvalue less than $\lambda^{*}$, by $\lambda_{k}$. If $\sigma_{0}$ in $\Sigma$ is such that Theorem 18 holds we use the notation $\lambda_{k}\left(\sigma_{0}\right)$ and $\lambda_{-k}\left(\sigma_{0}\right)$.

THEOREM 19. Let $\sigma_{0}$ in $\Sigma$ be given and assume $J\left(x ; \sigma_{0}\right)>0$ whenever $x \neq 0$ in $\mathfrak{A}\left(\sigma_{0}\right), K\left(x ; \sigma_{0}\right) \leqq 0$. Then there exists $\eta>0$ sush that $\rho\left(\sigma, \sigma_{0}\right)<\eta$ implies $J(x ; \sigma)>0$ whenever $x \neq 0$ in $\mathfrak{A}(\sigma), K(x ; \sigma)<0$.

In addition there exists $\lambda^{*}$ and $\delta>0$ such that $\mu=(\lambda, \sigma), \mu_{0}=$ $\left(\lambda^{*}, \sigma_{0}{ }^{\prime}, d\left(\mu, \mu_{0}\right)<\delta\right.$ imply $H(x ; \mu)>0$ on $\mathfrak{Y}(\mu)$.

If the first result is not true, we may choose sequences $\left\{\sigma_{q}\right\}$, $\left\{x_{q}\right\}$ such that $\sigma_{q} \rightarrow \sigma_{0}, \quad x_{q}$ in $\mathfrak{A}\left(\sigma_{q}\right),\left\|x_{q}\right\|=1, \quad K\left(x_{q} ; \sigma_{q}\right) \leqq 0$, and $J\left(x_{q} ; \sigma_{q}\right) \leqq 0$. Since $\left\{x_{q}\right\}$ is bounded there exist $y_{0}$ in $\mathfrak{A}$ and a subsequence $\left\{x_{q_{n}}\right\}$, which we assume to be $\left\{x_{q}\right\}$ such that $x_{q} \rightarrow y_{0}$. By (1) $y_{0}$ is in $\mathfrak{A}\left(\sigma_{0}\right)$.

We claim $y_{0}=0$. If not $K\left(y_{0} ; \sigma_{0}\right)=\lim _{q=\infty} K\left(x_{q} ; \sigma_{q}\right) \leqq 0$ implies $J\left(y_{0} ; \sigma_{0}\right)>0$ which is impossible as

$$
0 \geqq \limsup _{q=\infty} J\left(x_{q} ; \sigma_{q}\right) \geqq \liminf _{q=\infty} J\left(x_{q} ; \sigma_{q}\right) \geqq J\left(y_{0} ; \sigma_{0}\right) \text {. }
$$

Thus $J\left(y_{0} ; \sigma_{0}\right)=0=\lim _{q=\infty} J\left(x_{q} ; \sigma_{q}\right)$ and by $(2 \mathrm{c}) x_{q} \Rightarrow 0$. The contradiction $1=\lim _{q=\infty}\left\|x_{q}\right\|=\|0\|=0$ establishes the first result.

For the second result; by Theorem 18 there exists $\lambda^{*}$ such that $H\left(x ; \mu_{0}\right)>0$ on $\mathfrak{A}\left(\mu_{0}\right)$. Thus $n\left(\lambda^{*}, \sigma_{0}\right)=s\left(\lambda^{*}, \sigma_{0}\right)=0$. The result now follows by Corollary 17.

THEOREM 20. Let $\sigma_{0}$ in $\Sigma$ be given such that $J\left(x ; \sigma_{0}\right)>0$ whenever $x \neq 0$ in $\mathfrak{U}\left(\sigma_{0}\right), K\left(x ; \sigma_{0}\right) \leqq 0$. Assume $\lambda^{\prime}, \lambda^{\prime \prime}\left(\lambda^{\prime}<\lambda^{\prime \prime}\right)$ are not eigenvalues of $\sigma_{0}$ and there exists $k$ eigenvalues of $\sigma_{0}$ on $\left(\lambda^{\prime}, \lambda^{\prime \prime}\right)$. Then there exists $\varepsilon>0$ such that $\rho\left(\sigma, \sigma_{0}\right)<\varepsilon$ implies there are exactly $k$ eigenvalues of $\sigma$ on $\left(\lambda^{\prime}, \lambda^{\prime \prime}\right)$.

In fact if $\lambda_{n}\left(\sigma_{0}\right) \leqq \lambda_{n+1}\left(\sigma_{0}\right) \leqq \cdots \leqq \lambda_{n+k-1}\left(\sigma_{0}\right)$ are the $k$ eigenvalues of $\sigma_{0}$ on $\left(\lambda^{\prime}, \lambda^{\prime \prime}\right)$ then $\lambda_{n}(\sigma) \leqq \lambda_{n+1}(\sigma) \leqq \cdots \leqq \lambda_{n+k-1}(\sigma)$ are the $k$ eigenvalues of $\sigma$ on $\left(\lambda^{\prime}, \lambda^{\prime \prime}\right)$.

We may assume $\lambda^{*}\left(\sigma_{0}\right) \leqq \lambda^{\prime}<\lambda^{\prime \prime}$; if $\lambda^{\prime}<\lambda^{*}\left(\sigma_{0}\right)<\lambda^{\prime \prime}$ we consider the two intervals $\lambda^{\prime} \leqq \lambda \leqq \lambda^{*}\left(\sigma_{0}\right)$ and $\lambda^{*}\left(\sigma_{0}\right) \leqq \lambda \leqq \lambda^{\prime \prime}$ separately. Assume $s\left(\lambda^{\prime}, \sigma_{0}\right)=n$ then by Theorem 18, $s\left(\lambda^{\prime \prime}, \sigma_{0}\right)=n+k-1$, $n\left(\lambda^{\prime}, \sigma_{0}\right)=n\left(\lambda^{\prime \prime}, \sigma_{0}\right)=0$. By Corollary 14 there exists $\delta>0$ such that if $\rho\left(\sigma, \sigma_{0}\right)<\delta$ then $n\left(\lambda^{\prime}, \sigma\right)=n\left(\lambda^{\prime \prime}, \sigma\right)=0, s\left(\lambda^{\prime}, \sigma\right)=n$ and $s\left(\lambda^{\prime \prime}, \sigma\right)=$ $n+k-1$. The result follows from Theorem 18 by taking $\varepsilon=$ $\min (\delta, \eta)$ where $\eta$ given in Theorem 19. 
CoROLlary 21. If the $n$th eigenvalue $\lambda_{n}(\sigma)(n=0, \pm 1, \pm 2, \cdots)$ exists for $\sigma=\sigma_{0}$ it exists in a neighborhood of $\sigma_{0}$ and is a continuous function of $\sigma$.

We note that the continuity of the $n$th eigenvalue also follows from Theorem 11 as the hypothesis of Theorem 18 implies the hypothesis of Theorem 11.

Theorems 22 and 23 are concerned with comparison theorems and eigenvalue problem. These results have been given in Reference [2] and are included for completeness.

THEOREM 22. Let $\mathfrak{A}^{*}$ be a subspace of $\mathfrak{H}, J(x)>0$ whenever $x \neq 0$ and $K(x) \leqq 0$, and $\lambda^{*}$ be given as in Theorem 18. Let $\left\{\lambda_{i}\right\}$, $\left\{\lambda_{i}^{*}\right\}(i=0, \pm 1, \pm 2, \cdots)$ be the eigenvalues of $J(x)$ relative to $K(x)$ on $\mathfrak{U}$ and $\mathfrak{Q}^{*}$ respectively. If the $k$ th eigenvalues $\lambda_{k}$, $\lambda_{k}^{*}$ exist $(k=$ $0, \pm 1, \pm 2, \cdots)$ we have

$$
\begin{aligned}
& \lambda_{k} \leqq \lambda_{k}^{*} \quad(k=0,1,2, \cdots) \quad \text { and } \\
& \lambda_{k} \geqq \lambda_{k}^{*} \quad(k=-1,-2,-3, \cdots) .
\end{aligned}
$$

Strict inequality holds for any $k(k=0, \pm 1, \pm 2, \cdots)$ such that the $J\left(x ; \lambda_{k}\right)$ null vectors of $\mathfrak{A}$ and $\mathfrak{A}^{*}$ are disjoint.

If $\mathfrak{A} \ominus \mathfrak{H}^{*}$ has finite dimension e then

$$
\begin{aligned}
& \lambda_{k} \leqq \lambda_{k}^{*} \leqq \lambda_{k+e}(k=0,1,2, \cdots) \text { and } \\
& \lambda_{k} \geqq \lambda_{k}^{*} \geqq \lambda_{k-e}(k=-1,-2,-3, \cdots) .
\end{aligned}
$$

THEOREM 23. Let $J^{*}(x)$ and $K^{*}(x)$ be a second pair of elliptic and compact forms on $\mathfrak{A}$ such that $J^{*}(x)>0$ whenever $x=0$, $K^{*}(x)<0$. Let $J^{*}(x ; \lambda)=J^{*}(x)-\lambda K^{*}(x)$ and assume for any real $\lambda$ that $J(x ; \lambda) \leqq 0$ whenever $J^{*}(x ; \lambda) \leqq 0$. Then there exists $\lambda^{*}$ such that both $J^{*}\left(x ; \lambda^{*}\right)$ and $J\left(x ; \lambda^{*}\right)$ are positive definite on $\mathfrak{A}$.

Let $\left\{\lambda_{k}\right\}, \quad\left\{\lambda_{k}^{*}\right\}(k=0, \pm 1, \pm 2, \cdots)$ be the eigenvalues of $J(x)$ relative to $K(x)$ on $\mathfrak{U}$ and $J^{*}(x)$ relative to $K^{*}(x)$ on $\mathfrak{U}$ respectively. Then inequalities (13) hold. If $J(x ; \lambda)<0$ whenever $x \neq 0$ and $J^{*}(x ; \lambda)<0$ then inequalities (13) hold with strict inequality.

5. An Example. In this section we show that condition (1) and (2) include the hypothesis of the eigenvalue theory of $\mathrm{A}$. Weinstein [1]. Thus many physical problems, including those of vibrating membranes and plates, may be handled by our methods. In a subsequent paper we will indicate how the values $\lambda_{n}(\sigma)$ may be found by numerical methods.

The assumptions of Weinstein are now given. Gould [1] contains 
the most complete discussion of this theory as well as a complete list of references.

Let $\mathscr{L}$ be a closed subspace of $\mathfrak{A}$ and $\mathscr{L}^{n}(n=1,2, \cdots)$ be a sequence of closed subspaces of $\mathfrak{A}$. Let $P$ and $P^{n}$ be the respective projections of $\mathfrak{A}$ onto $\mathscr{C}$ and $\mathscr{L}^{n}$. The sequence $\left\{\mathscr{L}^{n}\right\}$ converges to $\mathscr{L}$, written $\mathscr{L}^{n} \rightarrow \mathscr{L}$ if $P^{n} x \Rightarrow P x$ for all $x$ in $\mathfrak{A}$. The sequence $\left\{\mathscr{L}^{n}\right\}$ converges downward to $\mathscr{L}$, written $\mathscr{L}^{n} \backslash \mathscr{L}$, if $\mathscr{L}^{n}$ converges to $\mathscr{L}$ and $\mathscr{S}^{n+1} \subset \mathscr{L}^{n}$. The sequence $\left\{\mathscr{L}^{n}\right\}$ converges upward to $\mathscr{L}$, written $\mathscr{L}^{n} \nearrow \mathscr{L}$, if $\mathscr{L}^{n}$ converges to $\mathscr{L}$ and $\mathscr{L}^{n} \subset \mathscr{L}^{n+1}$.

\section{Theorem 24. If $\mathscr{L}^{n} \searrow \mathscr{f}$ then $\mathscr{L}=\bigcap \mathscr{L}^{n}$. If $\mathscr{L}^{n} \nearrow \mathscr{L}$ then $\mathscr{L}=\overline{\mathrm{U} \mathscr{L}^{n}}$.}

If $\mathscr{L}^{n} \searrow \mathscr{L}$ then $x$ in $\mathscr{L}$ implies $x$ in $\mathscr{L}^{n}$ for $n=1,2, \ldots$ so that $x$ in $\bigcap \mathscr{L}^{n}$. Conversely if $x$ in $\mathscr{L}^{n}$ for each $n$ then $P^{n} x=x$ and hence $x=\lim _{n=\infty} P^{n} x=P x$ so that $x$ in $\mathscr{L}$.

If $\mathscr{L}^{n} \nearrow \mathscr{L}$ and $x$ not in $\mathscr{L}$ then $P x \neq x$. Thus there exists $\alpha>0$ such that

$$
\alpha<\|x-P x\|=\lim _{n=\infty}\left\|x-P^{n} x\right\|
$$

so that $x$ is not a limit point of $\mathrm{U} \mathscr{L}^{n}$ i.e., $x$ is not in $\overline{\mathrm{U} \mathscr{L}^{n}}$. Conversely if $x$ in $\mathscr{L}$ then $P^{n} x \Rightarrow P x=x$. Thus given $\varepsilon>0$ there exists $m$ such that $\left\|x-P^{m} x\right\|<\varepsilon$ with $P^{m} x$ in $\mathscr{L} \subset \bigcup \mathscr{L}^{n}$; hence $x$ is in $\overline{U \mathscr{L}^{n}}$.

A correspondence between Weinstein's setting and $\S 3$ is now given: Let $\Sigma=\left\{x \in E^{1} \mid x=1 / n(n=1,2, \cdots)\right.$ and 0$\}$ with the usual metric. For $n=1,2,3, \cdots$ we set $\mathfrak{A}(1 / n)=\mathscr{L}^{n}$ and $\mathfrak{A}(0)=\mathscr{L}$ where $\mathscr{L}, \mathscr{L}^{n}(n=1,2,3, \cdots)$ are subspaces of $\mathfrak{A}$ and $\mathscr{L}^{n} \nearrow \mathscr{L}$. A correspondence between the Generalized Raleigh-Ritz Method and $\S 3$ is obtained in the same manner except that $\mathscr{L}^{n} \searrow \mathscr{L}$.

Theorem 25. Assume $\left\{\mathscr{L}^{n}\right\}$ satisfies $\mathscr{L}^{n} \searrow \mathscr{L}$ or $\mathscr{L}^{n} \nearrow \mathscr{L}$. Then $n \rightarrow \infty, x_{n}$ in $\mathscr{L}^{n}, x_{n} \rightarrow y_{0}$ implies $y_{0}$ in $\mathscr{L}$.

Assume $\mathscr{L}^{n} \searrow \mathscr{L}$. Let $m$ be an arbitrary fixed integer. If $n \geqq m$ then $x_{n}$ in $\mathscr{L}^{m}$. It follows that $y_{0}$ is in $\mathscr{L}^{m}$. By Theorem 24, $y_{0}$ is in $\bigcap \mathscr{L}^{m}=\mathscr{L}$.

Assume $\mathscr{L}^{n} \nearrow \mathscr{L}$. By Theorem $24 \mathscr{L}=\overline{\mathrm{U} \mathscr{L}^{n}}$ and hence $x_{n}$ in $\mathscr{L}$ for $n=1,2, \cdots$. This implies, $y_{0}$ in $\mathscr{L}$.

Theorem 26. Assume $\left\{\mathscr{L}^{n}\right\}$ satisfies $\mathscr{L}^{n} \searrow \mathscr{L}$ or $\mathscr{L}^{n} \nearrow \mathscr{L}$. Then given any $x_{0}$ in $\mathscr{L}$ and $\varepsilon>0$ there exists a fixed integer $n_{0}$ such that if $n>n_{0}$ there exists $x_{n}$ in $\mathscr{L}^{n}$ satisfying $\left\|x_{0}-x_{n}\right\|<\varepsilon$. 
If $\mathscr{L}_{n} \searrow \mathscr{L}$ then $x_{0}$ in $\mathscr{L}=\bigcap \mathscr{C}^{n}$ and we take $x_{n}=x_{0}$ in $\mathscr{L}^{n}$ $(n=1,2, \cdots)$. If $\mathscr{L}^{n} \nearrow \mathscr{P}$ then $x_{0}$ in $\mathscr{L}=\overline{\mathrm{U} \mathscr{L}^{n}}$. Thus there exists $\bar{x}$ in $\bigcup \mathscr{L}^{n}$ such that $\left\|x_{0}-\bar{x}\right\|<\varepsilon$ and an $m$ such that $\bar{x}$ in $\mathscr{L}^{m}$. The result follows by taking $n_{0}=m$.

We remark that the spaces $\left\{\mathscr{C}^{n}\right\}$ are chosen by Weinstein in a more restrictive manner than that above. In particular for the case $\mathscr{L}^{n} \searrow$ they satisfy $\mathscr{L}^{n}=\mathscr{P}^{0} \ominus \operatorname{span}\left\{p_{1}, \cdots, p_{n}\right\}$ when $\left\{p_{k}\right\}$ is a complete orthonormal sequence in $\mathscr{L}^{0} \ominus \mathscr{L}$. In the case $\mathscr{P}^{n} \nearrow \mathscr{C}$ they satisfy $\mathscr{Z}^{n}=\mathscr{C}^{0} \oplus \operatorname{span}\left\{p_{1}, \cdots, p_{m}\right\}$ where $\left\{p_{k}\right\}$ is a complete orthonormal sequence in $\Theta \in$.

We note that inequalities (14) with $e=1$ include the comparison (or separation) results of Weinstein contained in [1; pp. 77].

\section{REFERENCES}

1. S. H. Gould, Variational Methods for Eigenvalue Problems, University of Toronto Press, Canada, 1966.

2. J. Gregory, An Approximation Theory for Elliptic Quadratic Forms on Hilbert Spaces, Dissertation, The University of California, Los Angeles, 1969.

3. M. R. Hestenes, Applications of the Theory of Quadratic Forms in Hilbert Space in the Calculus of Variations, Pacific J. Math. 1 (1951), 525-582.

4. A Weinstein, Separation Theorems for the Eigenvalues of Partial Differential Equations, Reissner Anniversary Volume, January, 1949.

Received April 10, 1970, and in revised form August 10, 1970. The preparation of this paper was sponsored in part by the U. S. Army Research Office under Grant DA-31-124-ARO(D)-355. Reproduction in whole or in part is permitted for any purpose of the United States Government.

University of CALIForNia, LOS ANGeles 



\section{PACIFIC JOURNAL OF MATHEMATICS}

\section{EDITORS}

\author{
H. SAMElson \\ Stanford University \\ Stanford, California 94305 \\ C. R. HOBBY \\ University of Washington \\ Seattle, Washington 98105
}

J. DugundjI

Department of Mathematics University of Southern California Los Angeles, California 90007

RICHARD ARENS

University of California

Los Angeles, California 90024

\section{ASSOCIATE EDITORS}
E. F. BECKENBACH
B. H. NeUmanN
F. WOLE
K. YOSHIDA

\section{SUPPORTING INSTITUTIONS}

\author{
UNIVERSITY OF BRITISH COLUMBIA \\ CALIFORNIA INSTITUTE OF TECHNOLOGY \\ UNIVERSITY OF CALIFORNIA \\ MONTANA STATE UNIVERSITY \\ UNIVERSITY OF NEVADA \\ NEW MEXICO STATE UNIVERSITY \\ OREGON STATE UNIVERSITY \\ UNIVERSITY OF OREGON \\ OSAKA UNIVERSITY \\ UNIVERSITY OF SOUTHERN CALIFORNIA
}

\author{
STANFORD UNIVERSITY \\ UNIVERSITY OF TOKYO \\ UNIVERSITY OF UTAH \\ WASHINGTON STATE UNIVERSITY \\ UNIVERSITY OF WASHINGTON \\ * * * \\ AMERICAN MATHEMATICAL SOCIETY \\ CHEVRON RESEARCH CORPORATION \\ NAVAL WEAPONS CENTER
}

The Supporting Institutions listed above contribute to the cost of publication of this Journal, but they are not owners or publishers and have no responsibility for its content or policies.

Mathematical papers intended for publication in the Pacific Journal of Mathematics should be in typed form or offset-reproduced, (not dittoed), double spaced with large margins. Underline Greek letters in red, German in green, and script in blue. The first paragraph or two must be capable of being used separately as a synopsis of the entire paper. The editorial "we" must not be used in the synopsis, and items of the bibliography should not be cited there unless absolutely necessary, in which case they must be identified by author and Journal, rather than by item number. Manuscripts, in duplicate if possible, may be sent to any one of the four editors. Please classify according to the scheme of Math. Rev. Index to Vol. 39. All other communications to the editors should be addressed to the managing editor, Richard Arens, University of California, Los Angeles, California, 90024.

50 reprints are provided free for each article; additional copies may be obtained at cost in multiples of 50 .

The Pacific Journal of Mathematics is published monthly. Effective with Volume 16 the price per volume (3 numbers) is $\$ 8.00$; single issues, $\$ 3.00$. Special price for current issues to individual faculty members of supporting institutions and to individual members of the American Mathematical Society: $\$ 4.00$ per volume; single issues $\$ 1.50$. Back numbers are available.

Subscriptions, orders for back numbers, and changes of address should be sent to Pacific Journal of Mathematics, 103 Highland Boulevard, Berkeley, California, 94708.

PUBLISHED BY PACIFIC JOURNAL OF MATHEMATICS, A NON-PROFIT CORPORATION

Printed at Kokusai Bunken Insatsusha (International Academic Printing Co., Ltd.), 7-17, Fujimi 2-chome, Chiyoda-ku, Tokyo, Japan. 


\section{Pacific Journal of Mathematics}

\section{Vol. 37, No. $2 \quad$ February, 1971}

Charles Compton Alexander, Semi-developable spaces and quotient images of metric spaces .................................... 277

Ram Prakash Bambah and Alan C. Woods, On a problem of Danzer. . . . . . . . . 295

John A. Beekman and Ralph A. Kallman, Gaussian Markov expectations and related integral equations . ....................................

Frank Michael Cholewinski and Deborah Tepper Haimo, Inversion of the Hankel

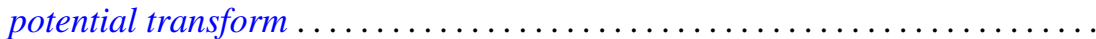

John H. E. Cohn, The diophantine equation

$$
Y(Y+1)(Y+2)(Y+3)=2 X(X+1)(X+2)(X+3) \ldots \ldots \ldots \ldots \ldots
$$

Philip C. Curtis, Jr. and Henrik Stetkaer, A factorization theorem for analytic

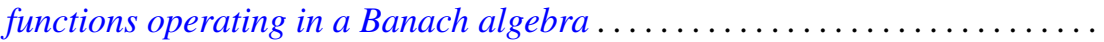

Doyle Otis Cutler and Paul F. Dubois, Generalized final rank for arbitrary limit ordinals

Keith A. Ekblaw, The functions of bounded index as a subspace of a space of entire functions

Dennis Michael Girard, The asymptotic behavior of norms of powers of

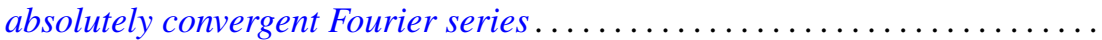

John Gregory, An approximation theory for elliptic quadratic forms on Hilbert spaces: Application to the eigenvalue problem for compact quadratic forms. 383

Paul C. Kainen, Universal coefficient theorems for generalized homology and stable cohomotopy.

Aldo Joram Lazar and James Ronald Retherford, Nuclear spaces, Schauder bases, and Choquet simplexes.

David Lowell Lovelady, Algebraic structure for a set of nonlinear integral operations

John McDonald, Compact convex sets with the equal support property . 429

Forrest Miller, Quasivector topologies

Marion Edward Moore and Arthur Steger, Some results on completability in commutative rings.

A. P. Morse, Taylor's theorem

Richard E. Phillips, Derek J. S. Robinson and James Edward Roseblade, Maximal subgroups and chief factors of certain generalized soluble groups.

Doron Ravdin, On extensions of homeomorphisms to homeomorphisms ...

John William Rosenthal, Relations not determining the structure of $\mathrm{L}$

Prem Lal Sharma, Proximity bases and subbases ........... .

Larry Smith, On ideals in $\Omega_{*}^{u}$. .

Warren R. Wogen, von Neumann algebras generated by operators similar to normal operators

R. Grant Woods, Co-absolutes of remainders of Stone-Čech 